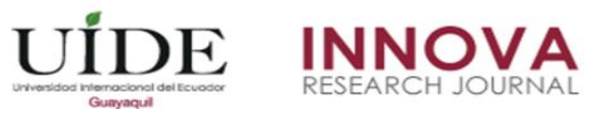

INNOVA Research Journal, ISSN 2477-9024

(Mayo, 2018). Vol. 3, No.5 pp. 54-64

DOI: https://doi.org/10.33890/innova.v3.n5.2018.522

URL: http://revistas.uide.edu.ec/index.php/innova/index

Correo: innova@uide.edu.ec

\title{
Intervención psicosocial preventiva de estrés laboral y abuso en el consumo de alcohol en una empresa venezolana
}

\section{Preventive psychosocial intervention of work stress and abuse in the consumption of alcohol in a venezuelan company}

Yolis Campos Villalta

Adonias Lubo Palma

Pamela Merino

Antonio Gómez

Universidad Internacional SEK Ecuador, Ecuador

Universidad del Zulia, Venezuela

Autor para correspondencia: yolis.campos@uisek.edu.ec

Fecha de recepción: 25 de Octubre de 2017 - Fecha de aceptación: 15 de Mayo de 2018

Resumen: Con el objetivo de prevenir, mediante intervención psicosocial, efectos negativos generados por el Estrés Laboral y los Trastornos por el Consumo de Alcohol, se realizó investigación descriptiva, longitudinal, de campo y correlacional en una empresa venezolana. La muestra estuvo constituida por 127 trabajadores del área operativa, a quienes se les aplicó el cuestionario de la Universidad Astral de Chile para Estrés Laboral y el test AUDIT para Consumo de Alcohol. Se identificaron 29 trabajadores con Estrés Laboral y 43 con trastornos por Consumo de Alcohol, respectivamente, de los que solo 11 exhibieron simultáneamente ambas variables, condición sine qua non para ejecutar la intervención. Además, a estos 11 trabajadores se les aplico el cuestionario CoPsoQ istas 21 y se realizó la intervención previa validación de expertos en el área, utilizando métodos expositivos y de discusión grupal e individual con la pertinente vigilancia y observación. Posteriormente, se evaluó el impacto correspondiente en los trabajadores. Los datos obtenidos se analizaron mediante la estadística descriptiva. En conclusión, el nivel de Estrés Laboral se redujo por cuanto las dimensiones Agotamiento Emocional y Despersonalización continuaron en el nivel bajo y la dimensión Logros personales se ubicó en el nivel medio. Con respecto a los Trastornos por Consumo de alcohol, se observó reducción en el consumo de riesgo y consumo perjudicial. Asimismo, se evidenció que las dimensiones exigencias psicosociales y doble presencia estuvieron dentro del nivel más favorable para la salud, la dimensión compensación en el nivel intermedio y el resto de las dimensiones apoyo social en la empresa y calidad de liderazgo y trabajo activo y posibilidades de desarrollo profesional evidenció un nivel más desfavorable para la salud. La relación entre las variables Estrés Laboral y Trastornos por el Consumo de Alcohol mostró una relación positiva, directa y débil.

Palabras Claves: estrés laboral; trastornos por el consumo de alcohol; dependencia de alcohol

Abstract: A descriptive, longitudinal, field and correlational investigation was carried out in the working population of a Venezuelan company. In order to prevent, through psychosocial intervention, negative effects generated by work stress and alcohol consumption abuse. The 
sample consisted of 127 workers from the operational area, who were given the questionnaire from the Universidad Astral de Chile for work stress and the AUDIT test. 29 workers with occupational stress and 43 with alcohol consumption abuse were identified, from which, only 11 exhibited both variables simultaneously, a sine qua non condition to perform the intervention. Therefore, the CoPsoQ 21 questionnaire was applied and the intervention was performed using expository methods as well as group and individual discussion with the relevant monitoring and observation. Once the intervention was completed, the corresponding impact on the workers was evaluated. Data were analyzed by using descriptive statistics. In conclusion, the level of work stress was reduced as the dimensions of emotional exhaustion and depersonalization continued at the low level and the personal achievement dimension was at the middle level. Regarding alcohol abuse, there was a reduction in the consumption of risk and harmful consumption. Likewise, CoPsoQ istas 21 showed that the dimensions of psychosocial requirements and double presence were within the most favorable level for health, the compensation dimension at the intermediate level and the rest of the dimensions social support in the company and quality of leadership and work active and professional development possibilities showed a more unfavorable level for health. The relationship between the variables labor stress and alcohol consumption was determined with the Spearman coefficient, whose results brought up 0.21, showing a direct, weak and positive relationship.

Key Words: work stress; alcohol abuse; alcohol dependency

\section{Introducción}

El mundo laboral es parte esencial del ser humano, influyendo en su salud y en su calidad de vida tanto en forma positiva como negativa (Blanco G. 2011, p. 235). La Organización Internacional del Trabajo (OIT) hoy en día reconoce que el entorno laboral puede ser un agente capaz de deteriorar la salud de las personas durante el desempeño de su trabajo, y que puede llegar a producir estrés, considerándolo un peligro para las economías de los países industrializados y en vías de desarrollo, ya que resiente la productividad, al afectar la salud física y mental de los trabajadores.

El Estrés Laboral constituye una de las enfermedades del siglo XXI, que afecta a millones de trabajadores en el mundo, independientemente del área que ocupe, debido a las grandes transformaciones en el mercado laboral, lo cual ha sido provocado por la globalización que impuso nuevas reglas en la competitividad de las empresas y organizaciones tanto para maximizar sus beneficios como por la necesidad de reducir costos, haciéndolas más rentables y productivas. De hecho, La Organización Mundial de la Salud (OMS) estima que entre el 5\% y el $10 \%$ de los trabajadores en los países industrializados padecen de Estrés Laboral, mientras que en los países en vías de desarrollo entre el 20\% y el 50\% los trabajadores estarían afectados por dicha enfermedad. (Mac Donald A. 2009).

Dentro de las condiciones sociales hay que destacar que el trabajo interviene positivamente en la salud, creando en las personas un sentimiento de identidad social, pero cuando este se desempeña en determinadas condiciones, como, por ejemplo, altos ritmos de trabajo, insatisfacción, entre otros, dichas condiciones pueden desencadenar el consumo de determinadas sustancias, como el alcohol (Prevención del consumo de alcohol en el lugar de trabajo. UGT. 2011, p. 3). 
Diversas hipótesis han sido generadas y contrastadas para abordar el consumo de alcohol, constituyendo el estrés una de las líneas más prolíferas y fecundas de investigación en relación al consumo de alcohol. La hipótesis que ha guiado a una gran parte de los estudios que vinculan el estrés con el consumo de alcohol es la llamada hipótesis de la reducción de tensión. Se asume que la ingesta de alcohol reduce la tensión o estrés psicológico y que cuando las personas se encuentran bajo estados de estrés, es más probable que consuman alcohol (Ergonomía y psicosociología aplicada. Edición $15^{\text {a }}$. 2009, vol. 1, Tema 8, pág. 457).

Los costos en el trabajo por la adicción al alcohol son significativos. Los expertos laborales estiman que el alcohol sigue siendo el mayor problema en el lugar de trabajo. En Estados Unidos existen más de diez (10) millones de alcohólicos, mientras que, en Europa, la ingestión de alcohol es un hábito profundamente involucrado en la sociedad (Weekly safety meetings. Vol.31. Issue 9. 2008).

Según la Organización Mundial de la Salud (OMS), de las diez (10) principales causas de discapacidad, cinco (5) de ellas corresponden a trastornos psiquiátricos, y dentro de estas se encuentra el alcoholismo, razón por la cual es un tema de interés (Diagnóstico y tratamiento en medicina laboral y ambiental. Joseph Ladou. 2006. Sección V. Capítulo 32, p. 628).

En la población latinoamericana no se tiene conocimiento preciso sobre la frecuencia de uso y abuso en el consumo de alcohol. Sin embargo, un estudio realizado por la Federación de Industrias del Estado de Sao Paulo (FIESP) sobre los problemas de la toxicomanía y el alcoholismo en el lugar de trabajo, señala que del 10 al 15\% de los empleados padecían problemas de adicción (Valencia J. y Pérez A. 2004, p. 3).

La OIT en febrero de 1998 creó un programa preventivo de intervención psicosocial con la finalidad de fomentar la adopción de nuevos planteamientos respecto al tratamiento de los problemas derivados del alcohol, basándose en una mayor participación del personal directivo en el lugar de trabajo y en la cooperación de los sindicatos, recurriendo al apoyo de los interlocutores sociales, con el fin de asegurar el acceso a las empresas y a su personal. Es un programa esencialmente preventivo que aborda la prevención del consumo de alcohol en el lugar de trabajo teniendo en cuenta las actitudes y los valores fomentados en la empresa, el entorno y las condiciones de trabajo (Revista de la OIT. Vol. 23.1998).

Una deficiente organización del trabajo, puede dar lugar a la aparición de riesgos psicosociales que repercuten directamente sobre la conducta de los trabajadores. Si se conocen estos factores y sus medidas preventivas, se podrá intervenir sobre estos y evitar los los ef $r$ que se producen, disminuyendo así las posibilidades del consumo de alcohol (Prevenciór consumo de alcohol en el lugar de trabajo. UGT. 2011, p. 38).

Internacionalmente existen varias organizaciones como la OIT (Revista de la OIT, $\mathrm{N}^{\circ} 30$, pp. 22-23), la OMS ( Serie Protección de la salud de los trabajadores Nº 3, 2004), Organización Panamericana de la Salud (Guía Internacional para vigilar el consumo de alcohol y sus consecuencias sanitarias. 2000), como entes privados y gobiernos que han promulgado programas y leyes en favor de la lucha contra los Riesgos Psicosociales; para la prevención y el manejo del Estrés Laboral y para el consumo de alcohol dentro de las empresas. 
En Venezuela, los Servicios de Seguridad y Salud en el Trabajo deben promover y mantener el nivel más elevado posible de bienestar físico, mental y social de los trabajadores, en tal sentido, es evidente que la intervención psicosocial forma parte del programa de vigilancia epidemiológica establecido en la Ley Orgánica de Prevención, Condiciones y Medio Ambiente de Trabajo (LOPCYMAT) y su Reglamento, en tal sentido en su artículo 53 establece: Los trabajadores (a) tendrán derecho a desarrollar sus labores en un ambiente de trabajo adecuado y propicio para el pleno ejercicio de sus facultades físicas y mentales y que garantice condiciones de seguridad, salud y bienestar adecuado.

Existen estudios de Estrés Laboral, especialmente en el sector salud, relacionadas con el alcohol en el lugar de trabajo; hay algunas investigaciones específicas que asocian al Estrés Laboral con el consumo de alcohol en el personal operativo de una empresa de dragado, sin embargo, carecen de programas que prevengan dichas patologías (Campos Y. 2012). Por lo tanto, el objetivo de este estudio es determinar el resultado de la intervención psicosocial preventiva de Estrés Laboral y el abuso en el consumo de alcohol en los trabajadores de una empresa venezolana.

\section{Metodología}

Se realizó un estudio descriptivo, longitudinal, de campo y correlacional en una empresa venezolana. La población objeto de estudio estuvo conformada por doscientos dos (202) trabajadores activos que se desempeñan como personal operativo, distribuidos de la siguiente manera: Pastificio (Empaque, Producción y Montacarguista), Molino (Empaque, Producción, Montacarguista y Recepción de Trigo), Mantenimiento (Electricistas, Mecánicos, Electromecánicos), Logística (Montacarguistas), Centro de Distribución (Operador de Almacén), Gerencia (Montacarguista) y Contraloría (Operadores de Balanza).

La muestra fue determinada por el criterio de Sierra Bravo y seleccionada de forma aleatoria por conglomerado, quedando conformada por 127 trabajadores con una antigüedad laboral igual o mayor a un año.

Al total de trabajadores que conformaron la muestra se les administró el cuestionario Autoaplicado de la Universidad Astral de Chile sobre Estrés Laboral y el Test de Identificación de Trastornos por Consumo de Alcohol (AUDIT), para determinar respectivamente Estrés Laboral y Abuso en el Consumo de Alcohol, estableciendo la correlación correspondiente mediante el coeficiente de Spearman y cuantificar el número de trabajadores que exhiban simultáneamente la presencia de ambas variables, requisito sine qua non para la ejecución de la correspondiente intervención psicosocial.

Del análisis por individuo se identificó a los trabajadores que refirieron Estrés Laboral, teniendo en cuenta los siguientes criterios: (1) nivel alto en cualquiera de las dimensiones; (2) trabajadores en los que se observó al menos dos niveles medios en cualquiera de las dimensiones. De acuerdo con el AUDIT, el análisis individual del Abuso en el Consumo del Alcohol se estableció como criterio de selección a aquellos individuos que reflejaran consumo de riesgo, consumo perjudicial y/o dependencia. 
A los trabajadores afectados se les realizó una intervención, la cual se llevó a cabo utilizando métodos expositivos, de discusión grupal e individual, métodos recreacionales y consultas multidisciplinarias; anticipadamente programados por el investigador y validados por expertos en el área, complementando dicha intervención con la correspondiente vigilancia y observación, previa aplicación del Cuestionario para la Evaluación de Riesgos Psicosociales en el Trabajo (CoPsoQ istas 21). Cumplida la intervención se aplica por segunda vez el Cuestionario Autoaplicado de la Universidad Astral de Chile sobre Estrés Laboral, el AUDIT y el CoPsoQ istas 21, para evaluar finalmente el impacto preventivo de la mencionada intervención psicosocial.

El análisis estadístico se realizó mediante la aplicación de la estadística descriptiva, mostrando las distribuciones de frecuencias absolutas y porcentuales. Del mismo modo, se utilizó el promedio de los datos, y la dispersión de los mismos se calculó mediante la desviación estándar con lo cual se establecieron los rangos en los cuales estaban las variables estudiadas en sus dimensiones.

\section{Resultados}

La Tabla 1 muestra la descripción general de la población. La edad promedio de la población trabajadora fue de 39 años, el 61,4\% estaba casada, el 63\% tenían entre 1 y 10 años de antigüedad, y el mayor porcentaje alcanzó el nivel de bachiller (44\%).

Tabla No. 1 Características sociodemográficas de la población de estudio

\begin{tabular}{|c|c|c|}
\hline & $(n=127)$ & $\%$ \\
\hline \multicolumn{3}{|l|}{ Edad } \\
\hline Límite Inferior & 23 años & \\
\hline Limite Superior & 58 años & \\
\hline Promedio & 39 años & \\
\hline \multicolumn{3}{|l|}{ Estado Civil } \\
\hline Casado & 78 & 61,4 \\
\hline Conviviente & 30 & 23,6 \\
\hline Soltero & 2 & 13 \\
\hline Divorciado & 17 & 2 \\
\hline Antigüedad (Años de servicio) & $\mathbf{N}^{\circ}$ & $\%$ \\
\hline De 1 a 10 & 80 & 63 \\
\hline De 11 a 20 & 31 & 24,4 \\
\hline De 21 a 30 & 15 & 11,5 \\
\hline De 31 a 38 & 1 & 1 \\
\hline \multicolumn{3}{|l|}{ Grado de Instrucción } \\
\hline Educación Primaria & 6 & 4,7 \\
\hline Educación Primaria Incompleta & 1 & 0,8 \\
\hline Bachiller & 56 & 44 \\
\hline Bachillerato incompleto & 10 & 8 \\
\hline Técnico Medio & 19 & 14,9 \\
\hline T.S.U. & 20 & 15,7 \\
\hline T.S.U. incompleto & 1 & 0,8 \\
\hline Universitario & 4 & 3,1 \\
\hline
\end{tabular}


En la Tabla 2 se presentan los resultados para la variable Estrés Laboral en sus tres dimensiones; agotamiento emocional, despersonalización y logros personales. En la dimensión Agotamiento Emocional aplicado al total de la muestra, luego en la pre intervención y post intervención. Se observa que, del total de 127 trabajadores, 76\% $(n=96)$ muestra un nivel bajo de Estrés Laboral, el 20\% ( $n=26)$ refleja un nivel medio y solo el 4\% (5 trabajadores) reflejan un nivel alto.

De los 127 trabajadores, solo 11 presentaron de forma simultánea Estrés Laboral y Abuso en el Consumo de Alcohol, por lo cual se les aplicó los instrumentos antes y después de la intervención. En la Pre intervención se observó que 73,0\% de los trabajadores (08), manifestaron un nivel bajo de Estrés Laboral en la dimensión Agotamiento Emocional; 18\% (02 individuos) reflejó un nivel medio y solo en el nivel alto, se evidencio el 9\% (01 individuo). El promedio fue de 13,5; con una desviación estándar de 4,9, lo que indica que la dimensión mencionada oscilo entre los puntajes de 8,6 y 18,4, es decir, entre los niveles bajo y medio con mayor tendencia hacia el nivel bajo.

Los resultados post intervención demuestran que el 64\% de los trabajadores (07) tienen un nivel bajo de Estrés Laboral en la dimensión Agotamiento Emocional; el 36\% (04 trabajadores) reflejó un nivel medio y el nivel alto no se evidenció. Por otro lado, el promedio fue de 13,5 con una desviación estándar de 2,0 lo que indica que la dimensión mencionada oscila entre los puntajes de 11,5 a 15,5. En la dimensión Despersonalización de la variable Estrés Laboral aplicado al total de la muestra, se observa que el 82\% $(n=104)$ de los trabajadores reflejó un nivel bajo de Estrés Laboral, mientras que el 13\% $(\mathrm{n}=17)$ alcanzó el nivel medio y el 5\% ( $\mathrm{n}=$ 6) se ubicó en el nivel alto. El promedio de los puntajes fue de 2,1 y la desviación estándar fue de 1,4; por lo cual la Despersonalización oscila entre los puntajes de 0,7 y 3,5 reflejando un nivel bajo.

Los resultados pre intervención en la dimensión Despersonalización muestran que $91 \%$ $(\mathrm{n}=10)$ reflejaron un nivel bajo de Estrés Laboral y 9\% $(\mathrm{n}=1)$ presentó un nivel alto. El promedio fue de 2,8 y la desviación estándar de 1,4; reflejando un nivel bajo. Posterior a la intervención, la dimensión Despersonalización refleja que el $73 \%(n=8)$ de los trabajadores presentaron un nivel bajo de Estrés Laboral, $18 \%(\mathrm{n}=2)$ resultaron con un nivel medio y solo $9 \%(\mathrm{n}=1)$ reportó nivel alto. El promedio de los puntajes fue de 3,1 y la desviación estándar de 0,5, observándose un comportamiento de la dimensión citada en el nivel bajo.

En cuanto a los resultados para la variable Estrés Laboral en la dimensión Logros Personales, 32\% (41) de los trabajadores evidenciaron un nivel bajo de Estrés Laboral, 48\% (61 individuos) se ubicaron en el nivel medio y $20 \%$ (25 trabajadores) en el nivel alto. El promedio obtuvo una puntuación de 36,9 y la desviación estándar fue de 2,8. Ubicando la dimensión citada en el nivel medio.

En cuanto a los resultados pre -intervención se observó que el 91\% (10) de los trabajadores encuestados quedaron ubicados en el nivel alto, el 9\% (01 trabajador), se ubicó en el 
nivel bajo. El promedio obtuvo una puntuación de 30,5; mientras que la desviación estándar fue de 12,7, lo que determinó que la dimensión se ubicara en el nivel alto.

Luego de la intervención 73\% (8) de los trabajadores reflejaron un nivel medio de Estrés Laboral en la dimensión Logros Personales, 18\% (02 trabajadores) resultaron con un nivel Bajo y solo un 9\% (01 individuo) reporto nivel Alto. El promedio fue de 36 y la desviación estándar fue de 1, reflejando un comportamiento de la dimensión citada en el nivel medio.

Tabla No. 2 Estrés Laboral

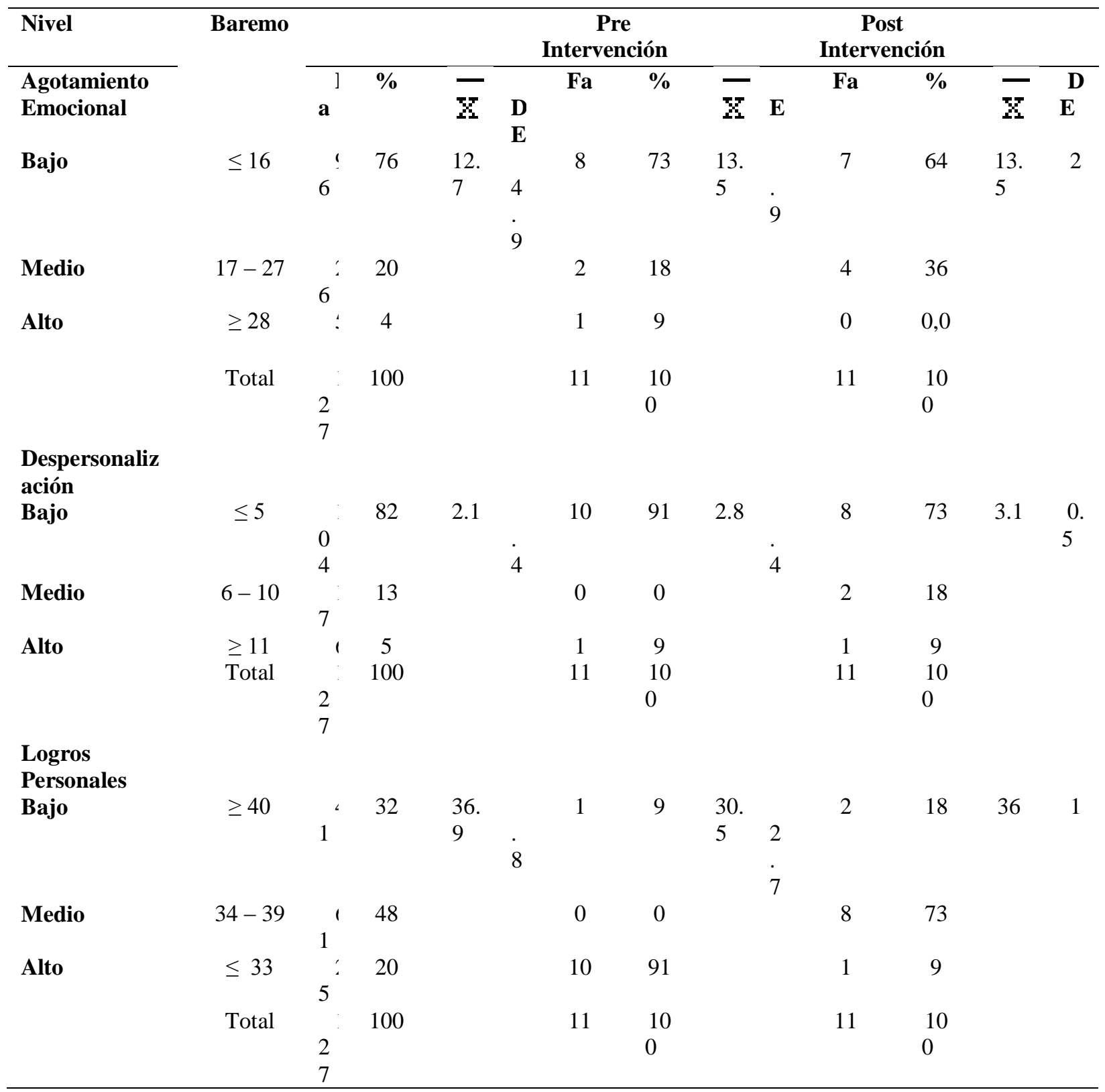

En la Tabla 3, se presentan los resultados para la variable Abuso en el Consumo de alcohol, donde el 66\% $(n=84)$ de los encuestados no reflejaron dificultades en el consumo; el 
29\% (37 sujetos) obtuvieron un consumo de riesgo; el 4\% (5 trabajadores), reportaron un consumo perjudicial y tan sólo un 1\% (1 trabajador) tuvo dependencia al alcohol.

Antes de la intervención, el $82 \%$ de los trabajadores (09) reflejaron consumo de riesgo; el $18 \%$ (02 individuos) tuvieron un consumo perjudicial y no se evidenció trabajadores con dependencia al alcohol. Al finalizar la intervención, el 27\% (03) de los trabajadores, no reflejaron dificultades en el consumo de alcohol, mientras que el 64\% (07 personas) presentó un consumo de riesgo y el 9\% (01 individuo) un consumo perjudicial. No se evidenció nivel de dependencia de alcohol en ningún trabajador.

Tabla No. 3 Abuso en el Consumo de alcohol

\begin{tabular}{|c|c|c|c|c|c|c|c|}
\hline \multirow[t]{2}{*}{ Nivel } & \multirow[t]{2}{*}{ Baremo } & \multicolumn{4}{|c|}{ Pre Intervención } & \multicolumn{2}{|c|}{$\begin{array}{c}\text { Post } \\
\text { Intervención }\end{array}$} \\
\hline & & $\mathbf{F a}$ & $\%$ & $\mathbf{F a}$ & $\%$ & $\mathbf{F a}$ & $\%$ \\
\hline Sin dificultad de Consumo & $<8$ & & & & & & \\
\hline Consumo de Riesgo al alcohol & $8-15$ & $\begin{array}{l}84 \\
37\end{array}$ & $\begin{array}{l}66 \\
29\end{array}$ & $\begin{array}{l}0 \\
9\end{array}$ & $\begin{array}{c}0 \\
82\end{array}$ & $\begin{array}{l}3 \\
7\end{array}$ & $\begin{array}{l}27 \\
64\end{array}$ \\
\hline Consumo Perjudicial al alcohol & $16-20$ & 5 & 4 & 2 & 18 & 1 & 9 \\
\hline Dependencia al alcohol. & $>20$ & 1 & 1 & 0 & 0 & 0 & 0 \\
\hline & 10 tal & 127 & 100 & 11 & 100 & 11 & 100 \\
\hline
\end{tabular}

En la Tabla 4 se muestran los resultados de la Evaluación de Riesgos Psicosociales presentes en el trabajo antes de la intervención, reflejando que el 91\% de los trabajadores tenían un nivel bajo en las exigencias psicológicas del trabajo, es decir, una condición favorable para la salud. Igualmente, las dimensiones compensaciones y doble presencia evidenciaron una condición favorable para la salud con un $73 \%$ y $55 \%$ respectivamente.

En cuanto al trabajo activo y posibilidades de desarrollo, así como el apoyo social en la empresa y calidad de liderazgo, alcanzaron ambas un $100 \%$ en el nivel de riesgo alto, es decir, en la condición más desfavorable para la salud.

Posterior a la intervención, las dimensiones exigencias psicológicas del trabajo y doble presencia reflejaron una condición más favorable para la salud de los trabajadores (riesgo bajo), obteniéndose un resultado de $100 \%$ y $64 \%$ respectivamente, mejorando la condición previa a la intervención.

En cuanto a las dimensiones, trabajo activo y posibilidades de desarrollo y apoyo social en la empresa y calidad de liderazgo, reportaron ambas una condición desfavorable para la salud de los trabajadores (riesgo alto) con un $100 \%$ cada una. Con respecto a la dimensión compensaciones, se observa que alcanzo valores similares de 45,5\% tanto para la condición más desfavorable para la salud de los trabajadores (riesgo alto) como para el nivel intermedio.

Tabla No. 4 Evaluación de Riesgos Psicosociales en el trabajo 


\begin{tabular}{|c|c|c|c|c|c|c|}
\hline \multirow{2}{*}{$\begin{array}{l}\text { DIMENSIÓN } \\
\text { SOCIAL }\end{array}$} & \multicolumn{3}{|c|}{ Pre Intervención } & \multicolumn{3}{|c|}{ Post Intervención } \\
\hline & $\begin{array}{c}\text { Bajo } \\
\text { (Más } \\
\text { favorable) }\end{array}$ & $\begin{array}{c}\text { Medio } \\
\text { (Intermedio) }\end{array}$ & $\begin{array}{c}\text { Alto } \\
\text { (Más } \\
\text { desfavorable) }\end{array}$ & $\begin{array}{c}\text { Bajo } \\
\text { (Más } \\
\text { favorable) }\end{array}$ & $\begin{array}{c}\text { Medio } \\
\text { (Intermedio) }\end{array}$ & $\begin{array}{c}\text { Alto(Más } \\
\text { desfavorable) }\end{array}$ \\
\hline $\begin{array}{l}\text { Exigencias } \\
\text { Psicológicas del }\end{array}$ & & $1(9 \%)$ & $0(0 \%)$ & $\begin{array}{c}11 \\
(100 \%)\end{array}$ & $0(0 \%)$ & $0(0 \%)$ \\
\hline Trabajo & $\begin{array}{c}10 \\
(91 \%)\end{array}$ & & & & & \\
\hline $\begin{array}{l}\text { Trabajo Activo } \\
\text { y Posibilidades } \\
\text { de Desarrollo }\end{array}$ & $0(0 \%)$ & $0(0 \%)$ & $11(100 \%)$ & $0(0 \%)$ & $0(0 \%)$ & $11(100 \%)$ \\
\hline $\begin{array}{l}\text { Apoyo Social en } \\
\text { la Empresa y } \\
\text { Calidad de } \\
\text { Liderazgo }\end{array}$ & $0(0 \%)$ & $0(0 \%)$ & $11(100 \%)$ & $0(0 \%)$ & $0(0 \%)$ & $11(100 \%)$ \\
\hline Compensaciones & $8(73 \%)$ & $3(27 \%)$ & $0(0 \%)$ & $1(9 \%)$ & $5(45,5 \%)$ & $5(45,5 \%)$ \\
\hline Doble Presencia & $6(55 \%)$ & $4(36 \%)$ & $1(9 \%)$ & $7(64 \%)$ & $4(36 \%)$ & $0(0 \%)$ \\
\hline
\end{tabular}

\section{Discusión}

Los resultados mostrados para la variable Estrés Laboral representan una condición favorable para los trabajadores, ya que la dimensión Agotamiento Emocional se mantuvo baja, la Despersonalización se redujo posterior a la intervención e igual comportamiento se observó en la dimensión Logros Personales.

Lo cual es congruente con los resultados encontrados por Campos Y. (2012), donde el personal operativo de una empresa de dragado arrojó bajos niveles de Estrés Laboral. Con respecto al Abuso en el Consumo de Alcohol, se encontró que la totalidad de la población intervenida presentó un consumo de riesgo y/o un consumo perjudicial. No obstante, con la aplicación del plan de intervención esta situación mejoró notablemente, pues el número de trabajadores con consumo de riesgo y consumo perjudicial se redujo, ubicándose en el renglón sin dificultad de consumo.

En este contexto, cabe destacar, que los resultados obtenidos en la empresa, antes de la intervención coinciden con los resultados planteados por Ramírez Danna y Col. (2011), quien afirma que existen Factores de Riesgo Psicosociales (organización del trabajo, factores individuales) que conducen al Consumo de Alcohol, reportándose ausentismo laboral, provocando accidentes, impidiendo el rendimiento en el trabajo. De allí puede observarse la relevancia que tuvo la aplicación del plan de intervención, donde al mejorar las condiciones, mejora también la vida laboral y familiar de los trabajadores en claro beneficio para la organización y el Estado.

Asimismo, los resultados obtenidos en relación al Abuso en el Consumo de Alcohol apoyan los resultados de la investigación realizada por González Elvira y Col. (2011), quien 
expone que los trabajadores con baja motivación y conflictos laborales tienen mayor probabilidad de consumición de riesgo de alcohol.

La investigación realizada por Carreño, Medina, Martínez y Col. (2006) confirma los resultados obtenidos en la etapa pre intervención en relación al Abuso en el Consumo de Alcohol, donde los trabajadores de una empresa textilera se ubican en un consumo perjudicial al aplicar el Cuestionario AUDIT, resultando que el Estrés Laboral es quien mejor predice el consumo de alcohol en el trabajo en relación al resto de los factores organizacionales considerados como son el clima organizacional y la satisfacción en el trabajo.

Este estudio tuvo como limitación que, en el momento de realizarse la intervención psicosocial a los trabajadores, los mismos se encontraban en discusión de contrataciones colectivas con la empresa, lo cual predispuso a los trabajadores intervenidos. Dentro de las fortalezas de la investigación debe resaltarse que nunca se había realizado un estudio de esa naturaleza en la empresa, lo cual servirá de guía para futuros estudios.

\section{Conclusiones}

Al comparar los resultados obtenidos de la medición del Estrés Laboral y el Abuso en el Consumo de Alcohol, se determinó que solo once (11) individuos exhibían en forma simultánea las dos variables. Razón por la cual, de acuerdo al objeto de la investigación, a estos trabajadores se les aplico las técnicas de intervención.

La relación entre las variables fue determinada mediante el Coeficiente de Spearman, cuyo resultado fue 0,21, lo cual indica que el Estrés Laboral influye directa y positivamente sobre el Abuso en el Consumo de Alcohol, pero con una intensidad que oscila entre muy débil y débil, pudiendo deducir que en el ambiente estudiado también pudieran estar presentes otros elementos que inciden sobre las variables mencionadas. Sin embargo, esta relación positiva justifica la intervención de los trabajadores afectados.

El mayor número de trabajadores con Estrés Laboral y el Abuso en el Consumo de Alcohol oscilan entre los 35 años y 50 años de edad, la mayoría son casados y con un grado de instrucción medio, ubicado entre bachiller y Técnico Superior Universitario.

Posterior a la intervención psicosocial se reporta un nivel aceptable de Estrés Laboral, reflejándose un nivel bajo con una leve tendencia al nivel medio para la dimensión Agotamiento Emocional. En cuanto a la dimensión Despersonalización, la misma se ubica en el nivel bajo, mientras que los Logros Personales se ubican en el nivel medio. En cuanto al Consumo de Alcohol, los trabajadores no reflejaron dificultades en el consumo, además se reduce el consumo de riesgo y el consumo perjudicial.

La exposición a los Riesgos Psicosociales en el trabajo reporta una condición más favorable para la salud en las dimensiones exigencias psicológicas y doble presencia, lo cual se traduce en un riesgo bajo. Las dimensiones, trabajo activo y posibilidades de desarrollo y apoyo social en la empresa y calidad de liderazgo, ambas se mantuvieron en alto riesgo, lo cual es una condición desfavorable para la salud de los trabajadores. Con respecto a la dimensión compensaciones, se 
observa que alcanzo valores similares tanto para la condición más desfavorable para la salud de los trabajadores como para el nivel intermedio.

\section{Bibliografía}

Abuso de alcohol y drogas en el trabajo (2008). Weekly safety meetings. Vol.31. Issue 9.

Blanco G. (2011). Estrés laboral, trabajo emocional y salud en profesionales del área de la rehabilitación. Revista Cubana de Salud y trabajo, vol. 12 (2), pp. 35.

Como combatir el estrés laboral. (2009). Manual Ibermutuamur. Editorial y Producción Arteanima, S.L, pp. 21.

Campo Y. (2012). Estrés Laboral y su relación con el consumo de alcohol en personal operativo de una empresa de dragado.

Constitución de la República Bolivariana de Venezuela. (2000).

Joseph Ladou. (4 edición). (2006). Diagnóstico y tratamiento en medicina laboral y ambiental. México. Manual Moderno. Sección V. Capítulo 32, p. 628.

Ley Orgánica de Prevención, Condiciones y Medio Ambiente de Trabajo. (2005). Caracas. Venezuela. Gaceta oficial No 38.236.

Llaneza J. (Edición 15ª). (2009). Ergonomía y psicosociología aplicada. Manejo para la formación del especialista. España. Editorial Lex Nova, vol. 1, Tema 8, pág. 457.

Mac Donald A. (2009). El estrés laboral en los países europeos y en América Latina. Consultoría abc Recursos Humanos.

Organización Internacional del Trabajo. (1999). El abuso de sustancias en el trabajo. Los sectores público y privado se alían contra el consumo de bebidas y drogas en el lugar de trabajo. Revista de la OIT, Nº 30, pp. 22-23.

Prevención del consumo de alcohol en el lugar de trabajo. (2011). Comisión ejecutiva confederal de UGT. Secretaria de Salud Laboral, 88, 28004-Madrid. Depósito legal: M 49908-2011, pp.3.

Valencia J. y Pérez A. (2004). Ausentismo laboral y su relación con el consumo de alcohol y otras sustancias psicoactivas (Protocolo), p. 3. 